\title{
Odevixibat: First Approval
}

\author{
Emma D. Deeks ${ }^{1}$
}

Published online: 9 September 2021

๑) Springer Nature 2021, corrected publication 2021

\begin{abstract}
Odevixibat (Bylvay ${ }^{\mathrm{TM}}$ ) is a small molecule inhibitor of the ileal bile acid transporter being developed by Albireo Pharma, Inc. for the treatment of various cholestatic diseases, including progressive familial intrahepatic cholestasis (PFIC). In July 2021, odevixibat received its first approval in the EU for the treatment of PFIC in patients aged $\geq 6$ months, followed shortly by its approval in the USA for the treatment of pruritus in patients aged $\geq 3$ months with PFIC. Odevixibat is also in clinical development for the treatment of other cholestatic diseases, including Alagille syndrome and biliary atresia, in various countries. This article summarizes the milestones in the development of odevixibat leading to this first approval for PFIC.
\end{abstract}

\section{Digital Features for this AdisInsight Report can be found at https:// doi.org/10.6084/m9.figshare.15184089.}

\section{Odevixibat (Bylvay $\left.{ }^{\mathrm{Tm}}\right)$ : Key points}

Small molecule inhibitor of the ileal bile acid transporter being developed by Albireo Pharma, Inc. for the treatment of various cholestatic diseases, including PFIC.

Received its first approval on 16 July 2021 in the EU.

Approved for the treatment of PFIC in patients aged $\geq 6$ months in the EU and for treatment of pruritus in patients aged $\geq 3$ months with PFIC in the USA.
This profile has been extracted and modified from the AdisInsight database. AdisInsight tracks drug development worldwide through the entire development process, from discovery, through preclinical and clinical studies to market launch and beyond.

Emma D. Deeks

dru@adis.com

1 Springer Nature, Mairangi Bay, Private Bag 65901, Auckland 0754, New Zealand

\section{Introduction}

Cholestatic liver diseases are typified by the accumulation of biliary components resulting from impaired bile formation and/or bile flow, with liver injury, inflammation and fibrosis being driven largely by bile acid (BA) retention [1, 2]. Symptoms can include pruritus, fatigue and jaundice, with progression to end stage liver disease in many cases requiring liver transplant [3]. Asymptomatic cases can also occur and are often identified by elevated levels of certain liver enzymes upon routine laboratory testing or tests for another condition [3].

Odevixibat (Bylvay ${ }^{\mathrm{TM}}$ ) is a small molecule inhibitor of the ileal BA transporter (IBAT) [4, 5]. Odevixibat is being developed by Albireo Pharma, Inc. (Albireo) for the treatment of cholestatic liver diseases, including progressive familial intrahepatic cholestasis (PFIC), biliary atresia and Alagille syndrome. In July 2021, odevixibat was approved in the EU for the treatment of PFIC in patients aged $\geq 6$ months $[5,6]$ and in the USA for the treatment of pruritus in patients aged $\geq 3$ months with PFIC [4, 7]. Odevixibat is available as 4 strengths in 2 different sized capsules; smaller capsules ( 400 and $1200 \mu \mathrm{g}$ ) are intended to be swallowed, while the larger capsules (200 and $600 \mu$ g strength) are intended to be opened and the contents (oral pellets) sprinkled on soft food $[4,5]$. The recommended dosage is $40 \mu \mathrm{g} / \mathrm{kg}$ once daily, administered orally in the morning, with (EU; USA) [4, 5] or without (EU) [5] food. In the 


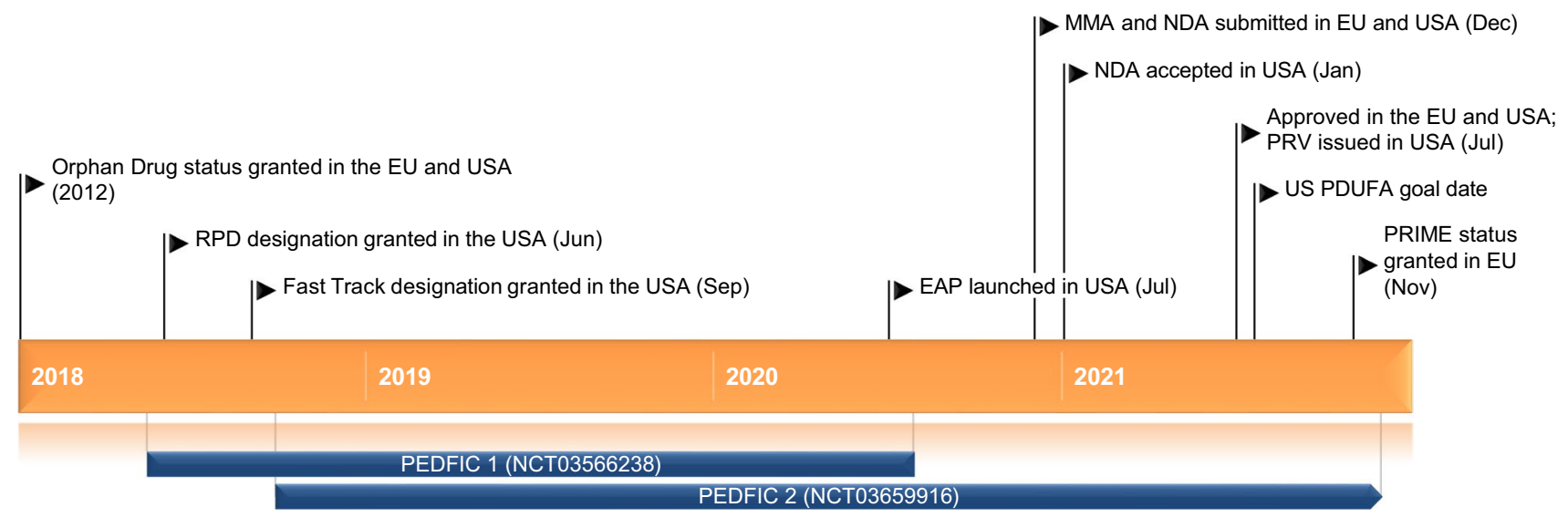

Key milestones in the development of odevixibat, focusing on its use in the treatment of progressive familial intrahepatic cholestasis. EAP Expanded Access Programme, MMA Marketing Authorization Appli-

EU and US, if an adequate clinical response has not been achieved after 3 months of continuous therapy with odevixibat, the dose may be increased to $120 \mu \mathrm{g} / \mathrm{kg} / \mathrm{day}[4,5]$; in the US this can be achieved by increasing the dose in increments of $40 \mu \mathrm{g} / \mathrm{kg}$ [4]. The maximum daily dose of odevixibat is $7.2 \mathrm{mg}$ per day in the EU [5] and the daily dose must not exceed $6 \mathrm{mg}$ in the US [4].

Odevixibat continues to be assessed for PFIC in an ongoing open-label, phase III extension study for long term safety and efficacy, and is also in phase III development for other cholestatic diseases, including Alagille syndrome and biliary atresia. Odevixibat has orphan designations for the treatment of Alagille syndrome, biliary atresia and primary biliary cholangitis in both the USA and EU [8-10].

\subsection{Company Agreements and Patent Information}

In February 2021, Albireo entered into a limited co-promotion agreement (for 2 years, with option of extending) with Travere Therapeutics in the USA [11] and entered a marketing agreement with Medison Pharma Ltd for commercialization of odevixibat in Israel [12]. In May 2021, Albireo also entered into license and distribution agreements for the commercialization of odevixibat with Genpharm Services (in Saudi Arabia and other Gulf countries) and Gen Ilac (in Turkey) [13].

In 2006, Albireo was issued patents covering the composition-of-matter for odevixibat in the USA and > 50 other countries, and in May 2017 was issued a method-of-treatment patent for odevixibat in PFIC and other specified liver diseases in the USA, corresponding method-of-use patents in Europe and Japan, and a method-of-treatment patent in NASH in the USA [14]. As of December 2020, Albireo has patent protection for odevixibat composition-of-matter expiring in September 2022, and protection in the USA for cation, NDA New Drug Application, PDUFA Prescription Drug User Fee Act, $P R I M E$ PRIority MEdicines, $P R V$ Paediatric Disease Priority Review Voucher, $R P D$ Rare Paediatric Disease

the method of using certain IBAT inhibitor(s) to treat certain liver diseases, expiring in November 2031, not including a patent term extension. Albireo also has a European patent for the method of using certain IBAT inhibitor(s) in combination with a BA binder to treat certain liver diseases (expires November 2031), with the same patent pending in the USA. An Albireo patent for crystal modifications of odevixibat is now issued in the USA (expiring in June 2039) and is currently pending in Europe. Similarly, patents for formulations of odevixibat are pending in the USA and Europe and, if granted, will expire in June 2039 [10].

\section{Scientific Summary}

\subsection{Pharmacodynamics}

Odevixibat displays selective, reversible inhibition of the IBAT [5]. The drug reduces the level of BAs in the plasma/ serum by reducing the reuptake of BAs in the distal ileum<smiles>CCCCC1(CCCC)CN(c2ccccc2)c2cc(SC)c(OCC(=O)N[C@H](C(=O)N[C@@H](CC)C(=O)O)c3ccc(O)cc3)cc2S(=O)(=O)N1</smiles>

Chemical structure of odevixibat 
and increasing colon clearance of BAs [5, 15]. There is no correlation between the degree by which serum BA (sBA) levels are reduced and the systemic pharmacokinetics of odevixibat, given the drug acts locally in the intestine with minimal systemic exposure [5].

In a mouse model of sclerosing cholangitis, odevixibat ameliorated cholestatic liver and bile duct injury and appeared to have anti-fibrotic as well as anti-inflammatory effects [16].

\subsection{Pharmacokinetics}

After oral administration, odevixibat absorption is minimal $[4,5]$. In paediatric patients with PFIC aged 0.5-17 years treated once daily with odevixibat 40 or $120 \mu \mathrm{g} / \mathrm{kg}$, plasma concentrations of odevixibat were generally below the limit of quantification [4]. Simulated in a paediatric PFIC population, odevixibat 40 or $120 \mu \mathrm{g} / \mathrm{kg} /$ day had maximum plasma concentrations of 0.21 and $0.62 \mathrm{ng} / \mathrm{mL}$ and area under the plasma concentration time-curve values of 2.26 and $5.99 \mathrm{ng} \cdot \mathrm{h} / \mathrm{mL}$ [5]. Taking odevixibat with food, or after sprinkling odevixibat pellets on soft food, has no clinically relevant impact on systemic exposure to the drug [4]. There is minimal [5] to no [4] accumulation of odevixibat after once-daily administration. Odevixibat is $>99 \%$ plasma protein bound [4, 5]. Dosages of 40 or $120 \mu \mathrm{g} / \mathrm{kg} / \mathrm{day}$ have mean bodyweight-adjusted apparent volumes of distribution of 40.3 and $43.7 \mathrm{~L} / \mathrm{kg}$ in paediatric patients [5]. Metabolism of odevixibat is minimal in humans [5], and occurs via mono-hydroxylation in vitro [4]. In healthy adults, elimination of odevixibat after a single oral dose occurred mainly via the faeces (82.9\%), with only $0.002 \%$ of the dose eliminated via the urine [17]. In paediatric patients who received odevixibat 40 or $120 \mu \mathrm{g} / \mathrm{kg} /$ day, the mean bodyweight-normalized apparent total clearance values were 26.4 and $23.0 \mathrm{~L} / \mathrm{kg} / \mathrm{h}$; the mean half-life of the drug is $\approx 2.5 \mathrm{~h} \mathrm{[5].}$

Absorption of fat-soluble vitamins (FSVs) may be reduced by odevixibat; monitoring of FSVs is therefore advised $[4,5]$. Similarly, odevixibat may impact the absorption of lipophilic oral contraceptives and other fatsoluble medications [5]. Odevixibat may be bound by BAbinding resins in the gut; thus, staggered administration is recommended [4]. Odevixibat is a P-glycoprotein substrate $[4,5]$; its exposure may therefore increase if coadministered with the strong P-glycoprotein inhibitor itraconazole, although this interaction is not expected to be clinically relevant $[18,19]$. Although odevixibat is an inhibitor of CYP3A4/5, it does not alter exposure to the CYP3A4 substrate midazolam $[4,5,19]$ or its 1 -hydroxy metabolite $[4$, 5] to any clinically relevant extent.

\subsection{Therapeutic Trials}

\subsubsection{Progressive Familial Intrahepatic Cholestasis}

Odevixibat was effective in reducing pruritus and sBAs in children with PFIC in a randomized, double-blind, phase III trial (NCT03566238; PEDFIC 1) [5, 20]. Patients aged 0.5-18 years with PFIC (type 1 or 2), elevated sBAs and a history of significant pruritus were randomized to odevixibat $40 \mu \mathrm{g} / \mathrm{kg}(n=23)$, odevixibat $120 \mu \mathrm{g} / \mathrm{kg}(n=19)$ or placebo $(n=20)$ once daily [20]. Over the 24-week treatment period, the mean proportion of positive pruritus assessments (defined as a scratching score of $\leq 1$ or $\geq 1$-point improvement from baseline on a 5-point scale of an observerreported instrument) [primary endpoint in USA] was

\section{Features and properties of odevixibat}

\begin{tabular}{|c|c|}
\hline Alternative names & Bylvay; A4250 \\
\hline Class & Acetamides, butyric acids, hepatoprotectants, small molecules, sulfones, thiazepines \\
\hline Mechanism of action & Sodium-bile acid cotransporter inhibitors \\
\hline Route of administration & Oral \\
\hline Pharmacodynamics & $\begin{array}{l}\text { Selectively and reversibly inhibits the ileal BA transporter; reduces BA levels in serum/plasma by reducing } \\
\text { BA reuptake in the distal ileum and increasing colon clearance of BAs }\end{array}$ \\
\hline Pharmacokinetics & Minimal absorption and metabolism; eliminated mainly via the faeces; half-life $\approx 2.5 \mathrm{~h}$ \\
\hline Most frequent adverse events & $\begin{array}{l}\text { Diarrhoea, increased hepatic enzymes (including alanine transferase and aspartate transferase), increased } \\
\text { blood bilirubin, increased international normalized ratio }\end{array}$ \\
\hline \multicolumn{2}{|l|}{ ATC codes } \\
\hline WHO ATC code & A05A-X05 (odevixibat) \\
\hline EphMRA ATC code & A5A (bile therapy and cholagogues) \\
\hline Chemical name & $\begin{array}{l}\text { (S)-2-((R)-2-(2-((3,3-dibutyl-7-(methylthio)-1,1-dioxido-5-phenyl-2,3,4,5-tetrahydrobenzo[f][1,2,5]thiadiaz- } \\
\text { epin-8-yl)oxy)acetamido)-2-(4-hydroxyphenyl)acetamido)butanoic acid }\end{array}$ \\
\hline
\end{tabular}

$B A$ bile acid 
$58.3 \%$ with odevixibat $40 \mu \mathrm{g} / \mathrm{kg} / \mathrm{day}, 47.7 \%$ with odevixibat $120 \mu \mathrm{g} / \mathrm{kg} /$ day and $28.7 \%$ with placebo, with the difference between each odevixibat group and placebo being significant (based on 95\% CIs) [5]. In addition, significantly (one-sided $p<0.02$ ) more patients achieved an sBA response (defined as a $\geq 70 \%$ reduction from baseline or a level $\leq 70 \mu \mathrm{mol} / \mathrm{L}$ at week 24) [primary endpoint in Europe/rest of world] with odevixibat $40 \mu \mathrm{g} / \mathrm{kg} /$ day $(43.5 \% ; 10 / 23$ patients) or $120 \mu \mathrm{g} / \mathrm{kg} /$ day $(21.1 \%$; $4 / 19$ patients) than with placebo (0\%; $0 / 20$ patients). Each odevixibat dosage group had favourable mean changes from baseline in growth parameters, including height and weight $\mathrm{Z}$ scores, versus placebo, although the between-group differences were not significant [5]. Odevixibat appeared to improve both patient and family healthrelated quality of life (QoL), as assessed by caregivers of patients aged $\geq 2$ years using the Pediatric QoL Inventory questionnaire, with mean improvements from baseline in both total score and family impact total score numerically favouring odevixibat (40 and $120 \mu \mathrm{g} / \mathrm{kg} /$ day pooled) over placebo [21].

Longer-term clinical benefit of odevixibat has been demonstrated in an ongoing, open-label, phase III, extension study (NCT03659916; PEDFIC 2) [22]. Patients in this trial either had PFIC type 1 or 2 and had been treated with odevixibat (40 or $120 \mu \mathrm{g} / \mathrm{kg} /$ day) $(n=34)$ or placebo $(n=$ $19)$ in the PEDFIC 1 trial or were new patients with any type of PFIC $(n=16)$. The previously treated patients were defined as the P1O cohort, whereas the latter two patient groups were combined into a single 'treatment-naïve' (TN) cohort. All patients received odevixibat $120 \mu \mathrm{g} / \mathrm{kg} / \mathrm{day}$ in the extension study. In an interim analysis (data cutoff July 2020), mean sBA levels in the $\mathrm{P} 1 \mathrm{O}$ cohort significantly $(p<0.0001)$ declined from $251.8 \mu \mathrm{mol} / \mathrm{L}$ at the PEDFIC 1 baseline to $85.1 \mu \mathrm{mol} / \mathrm{L}$ after 24 weeks of treatment in the extension (i.e. total of 48 weeks' therapy). In the P1O cohort, there were also significant improvements in mean monthly pruritus scores (from 3.0 to $1.4, p<0.0001$ ), height $\mathrm{Z}$ scores (from -1.6 to $-0.5, p=0.02$ ) and weight $\mathrm{Z}$ scores (from -0.9 to $0.2, p=0.03$ ) [22, 23]. Data for these outcomes in the TN cohort over the course of the extension (i.e. over 24 weeks' treatment) showed a similar trend [22, 23].

The efficacy of odevixibat has been further assessed in a pooled analysis of the PEDFIC 1 and 2 trials [24-26]. This analysis included 77 patients who received odevixibat in one or both of the studies; 42 were treated with the drug in PEDFIC 1 (34 of these entered PEDFIC 2), 19 had received placebo in PEDFIC 1 (all entered PEDFIC 2) and 16 were patients newly enrolled into PEDFIC 2 . In this analysis, improvements in sBA and pruritus were observed with odevixibat regardless of whether patients had type $1(n=20)$ or type $2(n=51)$ PFIC [24]; the improvements occurred within the first 4 weeks of treatment and were sustained up to week $48[25,26]$. In addition to improvements in sBA and pruritis, there were also continued clinically meaningful benefits seen in terms of growth, and sleep parameters over 48 weeks of treatment [26].

\subsubsection{Other Cholestatic Disease Studies}

Odevixibat demonstrated promise as a treatment for paediatric patients with various cholestatic liver diseases and pruritus in an open-label, multicentre, phase II study (NCT02630875) [27]. Eligible patients were aged 1-18 years with pruritus caused by a chronic cholestatic disease (e.g. PFIC, Alagille syndrome, biliary atresia or primary sclerosing cholangitis). Patients received a single dose of odevixibat (10-200 $\mu \mathrm{g} / \mathrm{kg}$, depending on the cohort) prior to a 2-week safety observation period, then received that same dose daily for 4 weeks; 20 patients participated, with 4 entering the study again after completing their first dose level. At the end of the 4-week treatment period, overall (i.e. all cohorts combined), the mean change from baseline in sBA

Key clinical trials of odevixibat (all sponsored by Albireo Pharma, Inc.)

\begin{tabular}{|c|c|c|c|c|c|}
\hline Drug(s) & Indication & Phase & Status & Location(s) & Identifier \\
\hline Odevixibat, placebo & PFIC (type 1 or 2 ) & III & Completed & Multinational & $\begin{array}{r}\text { NCT03566238; PEDFIC 1; } \\
\text { EudraCT2017-002338-21 }\end{array}$ \\
\hline Odevixibat & PFIC (any type) & III & Recruiting & Multinational & $\begin{array}{r}\text { NCT03659916; PEDFIC 2; } \\
\text { EudraCT2017-002325-38 }\end{array}$ \\
\hline Odevixibat, placebo & Alagille syndrome & III & Recruiting & Multinational & $\begin{array}{l}\text { NCT04674761; ASSERT; } \\
\text { EudraCT2020-004011-28 }\end{array}$ \\
\hline Odevixibat, placebo & Biliary atresia & III & Recruiting & Multinational & $\begin{array}{l}\text { NCT04336722; BOLD; } \\
\text { EudraCT2019-003807-37 }\end{array}$ \\
\hline Odevixibat & Cholestasis & II & Completed & Europe & $\begin{array}{l}\text { NCT02630875; } \\
\text { EudraCT2015-001157-32 }\end{array}$ \\
\hline
\end{tabular}

PFIC progressive familial intrahepatic cholestasis 
level (primary endpoint) was $-123.1 \mu \mathrm{mol} / \mathrm{L}(235 \mu \mathrm{mol} / \mathrm{L}$ at baseline). Mean changes from baseline in pruritus scores were -2.2 on the visual analogue-itch scale (baseline 6.2; $0-10$ scale), -2.0 on the Partial Patient-Oriented Scoring Atopic Dermatitis itch scale (baseline 6.0; 0-10 scale) and -0.8 on the Whitington itch scale (baseline $2.6 ; 0-4$ scale); lower scores indicate improvements [27].

\subsection{Adverse Events}

Odevixibat was generally well tolerated in paediatric patients with cholestatic liver diseases in clinical studies [20, 23, 27, 28], with the focus here being the phase III PEDFIC 1 and 2 trials.

In the 24-week PEDFIC 1 study, $33.3 \%$ of all odevixibat (40 or $120 \mu \mathrm{g} / \mathrm{kg} /$ day) recipients and $15.0 \%$ of placebo recipients had treatment-related adverse events (AEs) [28]. The most common of these to occur with odevixibat included diarrhoea, alanine aminotransferase (ALT) increased, blood bilirubin increased (incidence of each was $8.7 \%$ [2/23 patients] with $40 \mu \mathrm{g} / \mathrm{kg} / \mathrm{day}$ and $10.5 \%$ [2/19] with $120 \mu \mathrm{g} / \mathrm{kg} /$ day vs $5.0 \%$ [1/20] with placebo) and aspartate aminotransferase increased (8.7\% [2/23] with $40 \mu \mathrm{g} / \mathrm{kg} /$ day and $5.3 \%$ [1/19] with $120 \mu \mathrm{g} / \mathrm{kg} /$ day vs $5.0 \%$ [1/20] with placebo). One discontinuation occurred (in the odevixibat $120 \mu \mathrm{g} / \mathrm{kg} /$ day arm) because of an AE (diarrhoea) [28].

The tolerability profile of odevixibat in the PEDFIC 2 extension study was consistent with these findings. Interim tolerability data from PEDFIC 2 were grouped into two cohorts: patients who had received odevixibat $(n=34)$ or placebo $(n=19)$ in PEDFIC 1 (cohort 1) and patients who were newly enrolled into PEDFIC 2 (cohort 2; $n=$ 16) [28]. The incidence of treatment-related AEs with odevixibat $120 \mu \mathrm{g} / \mathrm{kg} /$ day from 24 weeks was $26.3-31.3 \%$ across cohorts 1 and 2 and 29\% (20/69 patients) overall. In cohort 1 the most common treatment-related AEs were blood bilirubin increased $(8.8 \% ; 3 / 34$ patients) and hepatic enzyme increased $(5.9 \% ; 2 / 34$ patients) in patients who had received odevixibat in PEDFIC 1, and blood bilirubin increased (10.5\%; $2 / 19$ patients) and ALT increased (5.3\%; $1 / 19$ patients) in patients who had received placebo in PEDFIC 1. Similarly, in cohort 2, blood bilirubin increased, ALT increased and international normalized ratio increased were the most frequent treatment-related AEs (12.5\% incidence for each; $2 / 16$ patients). One discontinuation occurred primarily because of an AE (worsening cholestasis) [28].

In PEDFIC 1 or 2 , there were no liver decompensation events, new FSV deficiency events (refractory to clinically recommended vitamin supplementation), deaths or serious treatment-related AEs, and no clinically relevant changes in haematology, serum biochemistry or urinalysis test values [28].

\subsection{Ongoing Clinical Trials}

In addition to the previously discussed ongoing, open label, long-term phase III PEDFIC 2 trial (NCT03659916) evaluating the safety and efficacy of odevixibat, recruitment is underway in two phase III trials evaluating the efficacy and safety of odevixibat in children and adults with Alagille syndrome (NCT04674761; ASSERT) and children with biliary atresia who have undergone a Kasai hepatoportoenterostomy (NCT04336722; BOLD).

\section{Current Status}

Odevixibat received its first approval on 16 July 2021 for the treatment of PFIC in patients aged $\geq 6$ months in the EU $[5,6]$ and on 20 July 2021 in the USA for the treatment of pruritus in patients aged $\geq 3$ months with PFIC $[4,7]$.

Supplementary Information The online version contains supplementary material available at https://doi.org/10.1007/s40265-021-01594-y.

\section{Declarations}

Funding The preparation of this review was not supported by any external funding.

Authorship and Conflict of Interest During the peer review process the manufacturer of the agent under review was offered an opportunity to comment on the article. Changes resulting from any comments received were made by the authors on the basis of scientific completeness and accuracy. Emma Deeks is a contracted employee of Adis International Ltd/Springer Nature, and declares no relevant conflicts of interest. All authors contributed to the review and are responsible for the article content.

Ethics approval, Consent to Participate, Consent to Publish, Availability of Data and Material, Code Availability Not applicable.

Open Access This article is licensed under a Creative Commons Attribution-NonCommercial 4.0 International License, which permits any non-commercial use, sharing, adaptation, distribution and reproduction in any medium or format, as long as you give appropriate credit to the original author(s) and the source, provide a link to the Creative Commons licence, and indicate if changes were made. The images or other third party material in this article are included in the article's Creative Commons licence, unless indicated otherwise in a credit line to the material. If material is not included in the article's Creative Commons licence and your intended use is not permitted by statutory regulation or exceeds the permitted use, you will need to obtain permission directly from the copyright holder. To view a copy of this licence, visit http://creativecommons.org/licenses/by-nc/4.0/.

\section{References}

1. Karpen SJ, Kelly D, Mack C, et al. Ileal bile acid transporter inhibition as anticholestatic therapeutic target in biliary atresia and other cholestatic disorders. Hepatol Int. 2020;14(5):677-89. 
2. de Vries E, Beuers U. Management of cholestatic disease in 2017. Liver Int. 2017;37:123-9.

3. European Association for the Study of the Liver. EASL clinical practice guidelines: management of cholestatic liver diseases. $\mathbf{J}$ Hepatol. 2009;51(2):237-67.

4. Albireo Pharma. Bylvay (odevixibat) capsules, for oral use: US prescribing information. 2021. https://albireopharma.com. Accessed 21 Jul 2021.

5. Albireo AB. Bylvay: EU prescribing information. 2021. https:// albireopharma.com. Accessed 21 Jul 2021.

6. FirstWorld Pharma. Albireo receives European marketing authorization of Bylvay (odevixibat), the first drug treatment for progressive familial intrahepatic cholestasis (PFIC) [media release]. 19 Jul 2021. https://www.firstwordpharma.com.

7. Albireo Pharma. Multimedia update-Albireo announces FDA approval of BylvayTM (odevixibat), the first drug treatment for patients with progressive familial intrahepatic cholestasis (PFIC) [media release]. $20 \mathrm{Jul}$ 2021. https://ir.albireopharma.com.

8. U.S. Food and Drug Administration. Orphan drug designations and approvals. 2021. https://www.accessdata.fda.gov/scripts/opdli sting/oopd/detailedIndex.cfm?cfgridkey $=658118$. Accessed 21 Jul 2021.

9. Albireo Pharma. Albireo granted orphan designation by European commission for lead product candidate A4250 for treatment of biliary atresia [media release]. 18 Dec 2018. http://www.albir eopharma.com.

10. United States Securities and Exchange Commission. Form 10-K. Albireo Pharma, Inc. 2021. https://www.sec.gov/Archives/edgar/ data/0001322505/000155837021001730/albo-20201231x10k. htm. Accessed 21 July 2021.

11. Albireo Pharma. Albireo reports Q4 and year-end 2020 financial results and business update [media release]. 25 Feb 2021. http:// www.albireopharma.com.

12. Albireo Pharma. Albireo presents odevixibat commercialization road to $\$ 1$ billion [media release]. 11 Feb 2021. www.albireopha rma.com.

13. Albireo Pharma. Albireo reports Q1 financial results and business update [media release]. 6 May 2021. http://www.albireopharma. com.

14. Albireo Pharma. Albireo announces two new U.S. patents allowed for A4250 with term into 2031 [media release]. 16 May 2017. http://www.albireopharma.com.

15. Graffner H, Gillberg PG, Rikner L, et al. The ileal bile acid transporter inhibitor A4250 decreases serum bile acids by interrupting the enterohepatic circulation. Aliment Pharmacol Ther. 2016;43(2):303-10.

16. Baghdasaryan A, Fuchs $\mathrm{CD}$, Österreicher $\mathrm{CH}$, et al. Inhibition of intestinal bile acid absorption improves cholestatic liver and bile duct injury in a mouse model of sclerosing cholangitis. J Hepatol. 2016;64(3):674-81.

17. Gillberg PG, Mattsson J, Torfgard K, et al. Clinical pharmacology of odevixibat, a potent, selective ileal bile acid transport inhibitor with minimal systemic exposure [abstract no. 167]. J Pediatric Gastroenterol Nutr. 2019;69(Suppl 2):113.

18. Stein P, Wright G, Kjems L, et al. Drug-drug interaction study to evaluate the interaction of A4250 (odevixibat) with itraconazole, a p-gp inhibitor in healthy adult subjects [abstract no. 638]. J Pediatric Gastroenterol Nutr. 2020;71(Suppl 1):S433-4.
19. Stein P, Wright G, Kjems L, et al. Drug-drug interaction study to evaluate the interaction of A4250 (odevixibat) with midazolam, a sensitive CYP3A4 substrate, and itraconazole, a P-gp inhibitor, in healthy adult subjects [abstract no. H-ePwP-033]. J Pediatric Gastroenterol Nutr. 2021;72(Suppl 1):873-4.

20. Thompson R, Baumann U, Czubkowski P, et al. Efficacy and safety of odevixibat, an ileal bile acid transporter inhibitor, in children with progressive familial intrahepatic cholestasis types 1 and 2: results from PEDFIC 1, a randomized, double-blind, placebo-controlled phase 3 trial [abstract no. LO4]. The Liver Meeting Digital Experience ${ }^{\mathrm{TM}}$. 2020. http://www.aasld.org/event/ liver-meeting-digital-experience. Accessed 21 Jul 2021.

21. Thompson RJ, Kjems L, Hardikar W, et al. Improved quality of life in children with progressive familial intrahepatic cholestasis following 24 weeks of treatment with odevixibat, an ileal bile acid transporter inhibitor: results from the phase 3 PEDFIC 1 study [abstract no. PMU62]. Value Health. 2021;24(Suppl 1):S155.

22. Thompson R, Artan R, D'Antiga L, et al. Long-term efficacy and safety of odevixibat, an ileal bile acid transporter inhibitor in children with progressive familial intrahepatic cholestasis: interim results from PEDFIC 2, an open-label phase 3 trial [abstract no. LP19]. The Liver Meeting Digital Experience ${ }^{\mathrm{TM}}$. 2020. http://www.aasld.org/event/ liver-meeting-digital-experience. Accessed 21 Jul 2021.

23. Thompson RJ, Verkade HJ, Gonzales E, et al. Long-term treatment benefits of odevixibat, an ileal bile acid transporter inhibitor, in children with progressive familial intrahepatic cholestasis: improvements in total bilirubin, growth, and sleep as secondary and exploratory outcome measures from the phase 3 PEDFIC 1 and PEDFIC 2 studies [abstract no. H-O-030]. J Pediatric Gastroenterol Nutr. 2021;72(Suppl 1):819-20.

24. Thompson R, Horn P, Houwen RHJ, et al. Substantial clinical benefits with odevixibat treatment across progressive familial intrahepatic cholestasis genetic deficiencies: subgroup analysis of serum bile acids, pruritus, and safety using pooled data from the PEDFIC 1 and 2 studies [abstract no. PO-1665 plus poster]. In: International Liver Congress. 2021.

25. Thompson R, D'Antiga L, Kamath BM, et al. Rapid and durable effect of odevixibat on clinical and biochemical parameters of cholestasis in children with progressive familial intrahepatic cholestasis [abstract no. OS-1554]. In: International Liver Congress. 2021.

26. Thompson R, D'Antiga L, Gonzales E, et al. Odevixibat therapy improves clinically meaningful endpoints in children with progressive familial intrahepatic cholestasis: data from the PEDFIC 1 and PEDFIC 2 trials [abstract no. PO-1833 plus poster]. In: International Liver Congress. 2021.

27. Baumann U, Sturm E, Lacaille F, et al. Effects of odevixibat on pruritus and bile acids in children with cholestatic liver disease: phase 2 study. Clin Res Hepatol Gastroenterol. 2021. https://doi. org/10.1016/j.clinre.2021.101751.

28. Grammatikopoulos T, Thompson C, Soufi N, et al. Long-term safety and tolerability of odevixibat, an ileal bile acid transporter inhibitor, in children with progressive familial intrahepatic cholestasis: results from the phase 3 PEDFIC 1 and PEDFIC 2 studies [abstract no. H-O-011]. J Pediatric Gastroenterol Nutrition. 2021;72(Suppl 1):781-2. 